\title{
Polarization Discrimination for Improving Foliage-Camouflaged Target Detection
}

\author{
Mojtaba Dehmollaian and Kamal Sarabandi \\ Radiation Laboratory \\ Department of Electrical Engineering and Computer Science \\ The University of Michigan, Ann Arbor, MI 48109-2122 \\ E-mail: saraband@eecs.umich.edu
}

\begin{abstract}
Many realizations of foliage around a hard target can be obtained using the coherent scattering model in a Monte Carlo simulation. These realizations provide the statistical variations of foliage and target response. Foliage camouflaged target detection at low microwave frequencies is investigated, using a powerful foliage and hard target simulation tool which accounts for near-field interactions between the hard target and foliage. Based on fully polarimetric simulation results of a foliage camouflaged metallic target a polarization synthesis optimization method for improving signal to clutter is examined. A genetic algorithm is implemented for finding an optimal set of radar transmit and receive polarizations that maximizes target backscatter to clutter ratio.
\end{abstract}

\section{INTRODUCTION}

A very comprehensive physics-based foliage and target scattering model has recently been developed that can account for the near field interactions among foliage, ground, and the target. In this model, individual trees in forest stand is decomposed into its basic constituents such as trunk, branches and leaves which are respectively modeled by dielectric cylinders and thin dielectric disks. Structure of trees are preserved using a statistical Lindenmayer system which can generate very complex tree structures. Next, single scattering theory, which also includes the interaction of the scatterers with the ground plane, is applied to compute the scattering from and propagation through the forest canopy. The ground plane is modeled by a dielectric half-space. Using this foliage model, a hybrid target-foliage model is then developed for investigating the scattering behavior of metallic hard targets embedded inside a forest canopy, at high frequencies [2]. This model is based on iterative physical optics (PO) approximation. Reciprocity theorem is used very effectively to compute the backscattered field using the incident fields, and induced electric currents on the target. This coherent scattering model can be run for many realizations of foliage around a hard target to obtain the statistical variations of foliage and target response in a Monte Carlo simulation. Foliage camouflaged target detection at low microwave frequencies (L-band) is investigated, using this powerful tool. Based on fully polarimetric simulation results performed at $2 \mathrm{GHz}$, for a metallic target embedded inside a pine forest stand a polarization synthesis optimization method for improving signal to clutter is examined. In this paper, we first summarize the backscattering computation formulation of metallic hard targets inside the foliage. Next a genetic algorithm is addressed for finding an optimal set of radar transmit and receive polarizations that maximizes target to clutter backscatter ratio.

\section{FORMULATION}

Using reciprocity theorem it can be shown that if the total incident electric field $\bar{E}_{1}$ and induced electric current $\bar{J}_{2}$ on a metallic target are known each component of the backscattered field can be computed from [2]

$$
\mathbf{S}_{p q}= \pm \frac{i k_{o} Z_{o}}{4 \pi} \int_{\mathbf{S}} \bar{E}_{1 p} \cdot \bar{J}_{2 q} d s^{\prime}
$$

where subscript $p$ and $q$ can be $h$ or $v$ for horizontal and vertical polarizations. The first 
and second subscripts denote the receive and the transmit polarizations, respectively. In (1), $\bar{E}_{1 p}$ is the $p$-component of the total incident field on the target, and $\bar{J}_{2 q}$ is the electric current on the surface of the hard target, induced by the $q-$ polarized incident wave. For problem at hand the total electric field inside the foliage $\bar{E}_{1}$ is coherent summation of all scatterers contribution in addition to the mean field, which has two components 1) direct 2) reflected from the ground plane. In the coherent scattering model each scatterer is illuminated by the mean field. Scattering from hard target itself can be computed using high frequency approximations. Due to the typical size of the hard targets, electrically large objects, PO approximation can provide very accurate scattered field near specular directions. In a forest with many scatterers around, many specular points can be obtained. In this approach the challenging problem is how to determine the shadow and lit regions on a complex target. This problem drastically exasperated for a target inside the forest being illuminated by a very large number of scatterers around. Thus, it is almost impossible to apply a ray tracing method. Noting that the shadowed regions are produced as a result of scattering in forward direction, we can implement an efficient iterative approach using the magnetic field integral (MFIE) equation, used to iterate on first order PO. The induced electric current $\bar{J}(\bar{r})$ on a metallic object, can be obtained from

$$
\begin{aligned}
& \bar{J}(\bar{r})= \\
& 2 \hat{n} \times \bar{H}_{i}(\bar{r})+ \\
& 2 \hat{n} \times \int_{\mathbf{S}}\left(\bar{r}-\bar{r}^{\prime}\right) \times \\
& \bar{J}\left(r^{\prime}\right)\left(i k_{0}-\frac{1}{\left|\bar{r}-\bar{r}^{\prime}\right|}\right) \frac{e^{i k_{0}\left|\bar{r}-\bar{r}^{\prime}\right|}}{4 \pi\left|\bar{r}-\bar{r}^{\prime}\right|^{2}} d \bar{r}^{\prime}
\end{aligned}
$$

It is noted that, if the surface of the object is smooth enough, the integral equation given by (2) can be solved iteratively. If the radii of curvature is small the integral part is small, therefore, to the first order approximation $\bar{J}^{(1)}=2 \hat{n} \times \bar{H}_{i}$, (the PO current is dominant). Higher order currents can be obtained using, $\bar{J}^{(m)}=2 \hat{n} \times L\left[\bar{J}^{(m-1)}\right]$, where $L\left[\bar{J}^{(m-1)}\right]$ is the surface integral evaluated for $(m-1)^{t h}$ order current $\bar{J}^{(m-1)}$. To calculate the field on the target for every source point, a simple geometrical optics (GO) shadowing condition given by $\hat{k} \cdot \hat{n}<0$ is used, where $\hat{k}$ is a unit vector from the source to the point on the surface, and $\hat{n}$ is a unit normal on the target. The same approach is implemented through the iteration. That is, in computation of (2), only the contribution of the points where,

$$
\left(\bar{r}-\bar{r}^{\prime}\right) \cdot \hat{n}<0
$$

is considered. This method, referred to as Hybrid GOPOPO, is applied to a target, shown in Fig. 1. The length of the target is $10 \lambda$ along $\hat{y}$ direction, and a radar operating frequency of $2 \mathrm{GHz}$ is assumed. Also the scattering problem is solved using MoM. A very good agreement is shown between exact electric field integral equation (EFIE) calculation, and the hybrid method. The advantage of this method becomes more clear when we put the object inside the forest canopy. In that case, for every scatterer and every point on the target only a simple condition $(\hat{k} \cdot \hat{n}<0)$ is checked where as for ray tracing approach every ray should be tracked. The iteration process on the other hand converges fast, and takes the effect of shadowing and multiple scattering on the target into account simultaneously.

\section{Simulation RESUlts}

The problem of particular interest is to discriminate the target backscattering response from that of the clutter. One way of achieving this, is to investigate the polarization responses of the foliage, and the foliage embedded target and examine whether there exists a particular set of transmit and receive polarizations that maximizes the signal to clutter in the statistical sense. An optimization procedure is needed to find the optimal radar polarization configuration for different clutter realizations and keeping the same target inside the clutter. Because of the discontinuous nature and the large number of local minima of the cost function a genetic algorithm (GA) is chosen for the optimization procedure. The advantage of GA is that it is not a gradient-based approach and the desired objective function is globally optimized. First we can search for a polarization 


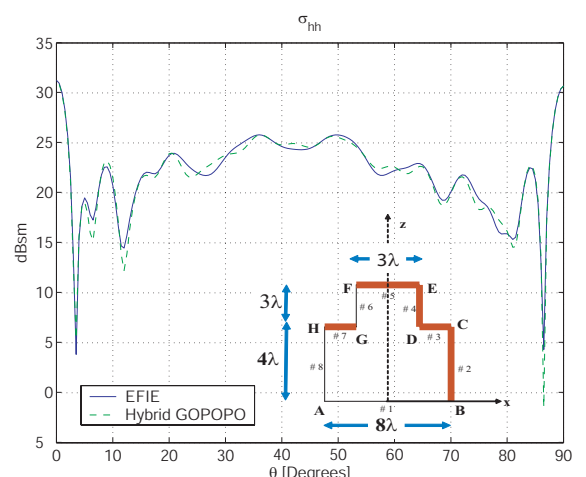

(a)

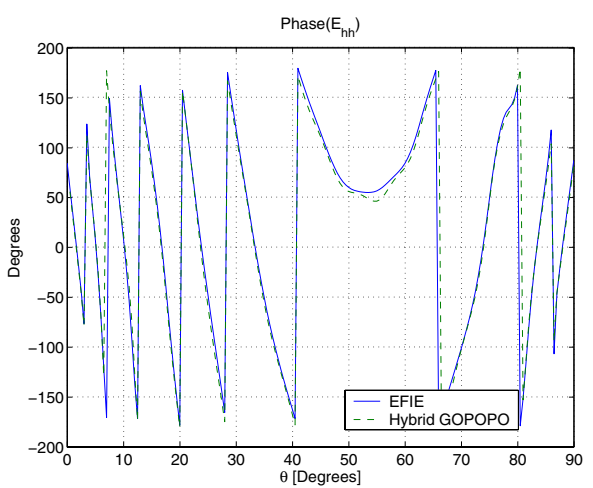

(b)

Fig. 1. Comparison of backscattering RCS calculated by exact EFIE method and Hybrid GOPO for a metallic target shown, at frequency of $2 \mathrm{GHz}, \phi=180^{\circ}$, and different elevation angles $\theta$. (a) $\sigma_{h h}$ (b) $\operatorname{Phase}\left(E_{h h}\right)$.

configuration that maximize the minimum signalto-clutter ratio, obtained by different realizations of the forest. Therefore the following optimization is presented,

$$
\begin{gathered}
\operatorname{Maximize}\left\{\operatorname{Min}\left\{\frac{\sigma_{t}}{\sigma_{c}}\left(\psi_{r}, \chi_{r}: \psi_{t}, \chi_{t}\right)\right\}_{m}\right\}_{p} \\
\forall m \in 1,2, \ldots, N_{R}
\end{gathered}
$$

subject to

$$
\psi_{r}, \psi_{t} \in\left[-90^{\circ}, 90^{\circ}\right] \quad \chi_{r}, \chi_{t} \in\left[-45^{\circ}, 45^{\circ}\right]
$$

where $m$ is the realization number, subscript $p$ stands for polarization and $N_{R}$ is the total number of realizations of the forest. $\psi$ and $\chi$ represent the tilt and ellipticity angles of polarization state, respectively. Therefore (4) provides a maximum level for signal-to-clutter ratio. However there might be situations where the response of the target is drastically distorted by the scattering from
TABLE I

THE OPTIMUM POLARIZATION STATES, DERIVED BY

GENETIC ALGORITHM USING 100 DIFFERENT SEEDS FOR INITIAL POPULATION SETS OF 200 CHROMOSOMES.

\begin{tabular}{|c|c|c|c|c|c|}
\hline & $\psi_{r}$ & $\chi_{r}$ & $\psi_{t}$ & $\chi_{t}$ & $\operatorname{Max}\{N\}$ \\
\hline \hline 1st Sol. & $38.95^{\circ}$ & $-8^{\circ}$ & $26.86^{\circ}$ & $-8^{\circ}$ & 71 \\
2nd Sol. & $-31.62^{\circ}$ & $-3^{\circ}$ & $-28.28^{\circ}$ & $6^{o}$ & 70 \\
\hline
\end{tabular}

the forest. Therefore by increasing the number of realizations, it is observed that the max-min value eventually approaches to zero. To solve this problem we maximize the number of cases where ratio of the backscatter RCS from embedded target to the backscatter RCS from the clutter is more than a specified level for all realizations of the forest. Accordingly, the objective function is

$$
\begin{gathered}
\text { Maximize }\left\{N\left[\frac{\sigma_{t}}{\sigma_{c}}\left(\psi_{r}, \chi_{r}: \psi_{t}, \chi_{t}\right)>L\right]_{m}\right\}_{p} \\
\forall m \in\left\{1,2, \ldots, N_{R}\right\}
\end{gathered}
$$

subject to (5). $N_{R}=100$ realizations of forest, consisting of 10 pine trees with density of 0.05 trees $/ \mathrm{m}^{2}$ is considered to examine the statistical variations of foliage and target response in a Monte Carlo simulation. Optimization (6) is performed for initial population of 200 chromosomes and different seeds $($ seed $=\{1,2, \ldots, 100\})$. Two states of polarization, achieved for $L=-8 d B$ is reported in table I. These optimum polarization configurations, provide about $70 \%$ of the times that we can have signal-to-clutter ratio of more than $-8 d B$. Physically, it is observed that transmit and receive polarizations are approximately perpendicular to the direction of tree branches and almost symmetric with respect to $\hat{v}$ direction.

\section{REFERENCES}

[1] Kamal Sarabandi, and Eric S. Li, Characterization of Optimum Polarization for Multiple Target Discrimination Using Genetic Algorithms, IEEE Trans. Antennas and Propagation. , Vol. 45, No. 12, December 1997.

[2] Mojtaba Dehmollaian and Kamal Sarabandi, A Forward Scattering Model for Foliage Camouflaged Complex Targets, Geoscience and Remote Sensing Symposium, 2004. IGARSS '04. Proceedings. 2004 IEEE International , 2024 Sep 2004. 